Moroccan J. of Pure and Appl. Anal. (MJPAA)

Volume 6(2), 2020, Pages 198-209

ISSN: Online 2351-8227 - Print 2605-6364

DOI: $10.2478 /$ mjpaa-2020-0015

\title{
Eigencurves of the $p(\cdot)$-Biharmonic operator with a Hardy-type term
}

\author{
Mohamed LAGHZAL ${ }^{1}$, Abdelouahed EL KHALIL ${ }^{2}$, My Driss MORCHID ALAOUI ${ }^{3}$, AND \\ AbDelfattah TOUZANI 4
}

Aвstract. This paper is devoted to the study of the homogeneous Dirichlet problem for a singular nonlinear equation which involves the $p(\cdot)$-biharmonic operator and a Hardy-type term that depend on the solution and with a parameter $\lambda$. By using a variational approach and min-max argument based on LjusternikSchnirelmann theory on $C^{1}$-manifolds [13], we prove that the considered problem admits at least one nondecreasing sequence of positive eigencurves with a characterization of the principal curve $\mu_{1}(\lambda)$ and also show that, the smallest curve $\mu_{1}(\lambda)$ is positive for all $0 \leq \lambda<C_{H}$, with $C_{H}$ is the optimal constant of Hardy type inequality.

Mathematics Subject Classification (2010). 58E05, 35J35, 35J60, 47J10.

Key words and phrases. $p(\cdot)$-biharmonic operator, Variational methods, Ljusternik-Schnirelmann theory, $p(\cdot)$-Hardy's inequality.

Received 11 May 2020 - Accepted: 06 July 2020.

(C) This article is published with open access by Sidi Mohamed Ben Abdallah University.

The Authors: 1, 4 Laboratory LAMA Department of Mathematics Faculty of Sciences Dhar El Mahraz University Sidi Mohamed Ben Abdellah P.O. Box 1796 Atlas Fez Morocco.

e-mail: ${ }^{1}$ mohamed.laghzal@usmba.ac.ma (Corresponding author)

e-mail: ${ }^{4}$ Abdelfattah.touzani@usmba.ac.ma

${ }^{2}$ Department of Mathematics and Statistics College of Science Imam Mohammad Ibn Saud Islamic University (IMSIU) P.O. Box 90950, 11623 Riyadh KSA.

e-mail: 2elakhalil@imamu.edu.sa

${ }^{3}$ AMNEA Group Department of Mathematics Faculty of Sciences and Technologies Moulay Ismail University BP 509 Boutalamine 52000 Errachidia Morocco.

e-mail: ${ }^{3}$ morchid.driss@yahoo.fr . 


\section{Introduction}

Nonlinear singular elliptic problems have been studied intensively in recent years, we mention the work [8]. For instance, nonlinear singular boundary value problems arise in some parts of science such as in boundary layer phenomena for viscous fluids, chemical heterogeneous catalysts, chemical catalyst kinetics, and theory of heat conduction in electrically conducting materials.

Hardy inequality in $W_{0}^{2, p}(\Omega)$ and $W_{0}^{2, p(\cdot)}(\Omega)$ and the related problems for nonlinear elliptic equation have attracted considerable interest. We refer to papers [6, 7, 9], where further bibliographical references can be found.

Motivated by such interest, we consider the singular $p(\cdot)$-biharmonic problem

$$
\left(E_{\lambda}\right) \begin{cases}\Delta\left(|\Delta u|^{p(x)-2} \Delta u\right)-\lambda \frac{|u|^{p(x)-2} u}{\delta(x)^{2 p(x)}}=\mu|u|^{p(x)-2} u, & \text { for } x \in \Omega, \\ u=0, & \text { for } x \in \partial \Omega\end{cases}
$$

where $\Omega$ is a regular bounded domain in $\mathbb{R}^{N}, N \geq 3, \delta(x):=\operatorname{dist}(x, \partial \Omega)$ denotes the distance from $x$ to the boundary $\partial \Omega$. Throughout this paper the function $p \in C(\bar{\Omega})$ always denotes a variable exponent satisfying the following bounds

$$
1<p^{-}:=\min _{\bar{\Omega}} p \leq p^{+}:=\max _{\bar{\Omega}} p<\frac{N}{2},
$$

here $\mu$ plays the role of an eigenvalue depending on $\lambda$ (referred as eigencurve $: \lambda \mapsto \mu(\lambda)$ ), and $\lambda$ is a real parameter such that $0 \leq \lambda<C_{H}$, where

$$
C_{H}:=\min \left(\frac{p^{-}}{p^{+}} \gamma_{1}^{p^{-}} ; \frac{p^{-}}{p^{+}} \gamma_{2}^{p^{+}}\right),
$$

is a positive constant in the $p(\cdot)$-Hardy inequality, with

$$
\gamma_{1}=\left[\frac{N\left(p^{-}-1\right)\left(N-2 p^{-}\right)}{\left(p^{-}\right)^{2}}\right] \text { and } \gamma_{2}=\left[\frac{N\left(p^{+}-1\right)\left(N-2 p^{+}\right)}{\left(p^{+}\right)^{2}}\right],
$$

and the $p(\cdot)$-Hardy inequality [6, Lemma 3.1] is given by

$$
\int_{\Omega} \frac{1}{p(x)}|\Delta u|^{p(x)} d x \geq C_{H} \int_{\Omega} \frac{1}{p(x)} \frac{|u|^{p(x)}}{\delta(x)^{2 p(x)}} d x,
$$

for all $u \in W_{0}^{2, p(\cdot)}(\Omega)$.

$\Delta_{p(\cdot)}^{2} u:=\Delta\left(|\Delta u|^{p(x)-2} \Delta u\right)$ is the $p(\cdot)$-biharmonic operator. It is reduced to the $p$-biharmonic (for a constant exponent $p>1$ ). For variable exponent the $p(\cdot)$-biharmonic presents a more complicated nonlinearity than the $p$-biharmonic, we loose homogenity of any order.

In [6], the case $\mu=0$ was considered. By the Ljusternik-Schnirelmann theory on $C^{1}$ manifolds $[13,4]$, the existence of a sequence of positive eigenvalues was proved. Let $\Lambda$ be the set of all nonnegative eigenvalues. It was shown that $\sup \Lambda=\infty$. Sufficient conditions were also found to guarantee that inf $\Lambda>0$. 
The eigencurve problems involving the $p$-Laplacian operator has been the object of study in [2]. Precisely, the authors have studied the problem $-\Delta_{p} u-\lambda \rho(x)|u|^{p-2} u=\mu|u|^{p-2} u$, on a bounded regular domain.

In [5], without any assumption on the regularity of the bounded domain $\Omega$, the authors have studied the problem $\Delta_{p}^{2} u-\lambda \rho(x)|u|^{p-2} u=\mu|u|^{p-2} u$. Using the variational methods, they obtained the existence of an increasing sequence of positive eigencurves.

The goal of this paper is to show that problem $\left(E_{\lambda}\right)$ has at least one non-decreasing sequence of eigencurves $\left(\mu_{k}(\lambda)\right)_{k \geq 1}$, as we give a direct characterization of the principal curve, by using a variational technique based on Ljusternik- Schnirelmann theory on $C^{1}$-manifolds [13].

The paper is organized as follows. In the second section, we provide a suitable functional framework for problem $\left(E_{\lambda}\right)$ and prove some preliminary results. In the third section, we prove the compactness of the functional $\Phi_{\lambda}$ associated with $\left(E_{\lambda}\right)$.

Finally, in the fourth Section, we obtain the existence of an increasing sequence of positive eigencurves of the $p(\cdot)$-biharmonic operator with a Hardy-type term.

\section{Functional framework and preliminaries}

In this section, we briefly review the definitions and list some results on the spaces $L^{p(\cdot)}(\Omega)$ and $W^{m, p(\cdot)}(\Omega)$, where $\Omega$ is given as in problem $\left(E_{\lambda}\right)$. We refer the reader to the book [3] and the references therein, for the basic properties of these spaces.

Let

$$
C_{1}^{+}(\bar{\Omega}):=\{h \mid h \in C(\bar{\Omega}) \text { and } h(x)>1 \text { on } \bar{\Omega}\} .
$$

Let $p \in C_{1}^{+}(\bar{\Omega})$ be fixed. We define the generalized Lebesgue space by

$$
L^{p(\cdot)}(\Omega):=\left\{u \mid u: \Omega \rightarrow \mathbb{R} \text { measurable and } \rho_{p(.)}(u)<\infty\right\},
$$

endowed with the so-called Luxemburg norm

$$
|u|_{p(\cdot)}:=\inf \left\{\alpha>0 \mid \rho_{p(\cdot)}\left(\frac{u}{\alpha}\right) \leq 1\right\},
$$

where $\rho_{p(\cdot)}: L^{p(\cdot)}(\Omega) \rightarrow \mathbb{R}$ is the convex modular mapping defined by

$$
\rho_{p(\cdot)}(u)=\int_{\Omega}|u|^{p(x)} d x .
$$

We define the potential

$$
\Psi_{p(.)}(u)=\int_{\Omega} \frac{1}{p(x)}|u|^{p(x)} d x
$$


Proposition 2.1 (see [14]). Under hypothesis (1.1), the space $\left(L^{p(\cdot)}(\Omega),|\cdot|_{p(.)}\right)$ is separable, uniformly convex, reflexive and its conjugate dual space is $L^{p^{\prime}(\cdot)}(\Omega)$ where

$$
p^{\prime}(x)=\frac{p(x)}{p(x)-1} \text { for all } x \in \Omega .
$$

For $u \in L^{p(\cdot)}(\Omega)$ and $v \in L^{p^{\prime}(\cdot)}(\Omega)$ we have the Hölder-type inequality

$$
\left|\int_{\Omega} u(x) v(x) d x\right| \leq\left(\frac{1}{p^{-}}+\frac{1}{p^{\prime-}}\right)|u|_{p(\cdot)}|v|_{p^{\prime}(\cdot)} \leq 2|u|_{p(\cdot)}|v|_{p^{\prime}(\cdot)} .
$$

The Sobolev space with variable exponent $W^{m, p(\cdot)}(\Omega)$, where $m$ is a positive integer, is defined as

$$
W^{m, p(\cdot)}(\Omega):=\left\{u \in L^{p(\cdot)}(\Omega)\left|D^{\alpha} u \in L^{p(\cdot)}(\Omega),\right| \alpha \mid \leq m\right\} .
$$

Equipped with the norm

$$
\|u\|_{m, p(\cdot)}:=\sum_{|\alpha| \leq m}\left|D^{\alpha} u\right|_{p(\cdot)} .
$$

$W^{m, p(\cdot)}(\Omega)$ are Banach, separable and reflexive spaces. For more details, we refer the reader to $[10,11,12]$ and the references therein. $W_{0}^{m, p(\cdot)}(\Omega)$ is denoted by the closure of $C_{0}^{\infty}(\Omega)$ in $W^{m, p(\cdot)}(\Omega)$.

Proposition 2.2 (see [11, Theorem 1.3]). Let $u_{n}, u \in L^{p(\cdot)}(\Omega)$, we have

1. $|u|_{p(\cdot)}=a \Leftrightarrow \rho_{p(.)}\left(\frac{u}{a}\right)=1$ for $u \neq 0$ and $a>0$.

2. $|u|_{p(.)}<(=;>1) \Leftrightarrow \rho_{p(.)}(u)<(=;>1)$.

3. $\left|u_{n}\right| \rightarrow 0(\operatorname{res} p \rightarrow+\infty) \Leftrightarrow \rho_{p(.)}\left(u_{n}\right) \rightarrow 0,(\operatorname{res} p \rightarrow+\infty)$.

4. The following statements are equivalent to one another:

(i) $\lim _{n \rightarrow+\infty}\left|u_{n}-u\right|_{p(\cdot)}=0$,

(ii) $\lim _{n \rightarrow+\infty} \rho_{p(.)}\left(u_{n}-u\right)=0$,

(iii) $u_{n} \rightarrow u$ in measure in $\Omega$ and $\lim _{n \rightarrow+\infty} \rho_{p(.)}\left(u_{n}\right)=\rho_{p(.)}(u)$.

Throughout this paper, we let $X:=W_{0}^{2, p(\cdot)}(\Omega)$, and $X^{*}:=W^{-2, p^{\prime}(\cdot)}(\Omega)$ its dual. Define a norm $\|\cdot\|$ of $X$ by

$$
\|u\|:=\inf \left\{\alpha>0 \mid \rho_{p(.)}\left(\frac{\Delta u}{\alpha}\right) \leq 1\right\} .
$$

Remark 2.1. It easy to see that $\|\cdot\|_{2, p(\cdot)}$ is equivalent to the norm $|\Delta(.)|_{p(\cdot)}$ in the space X. Consequently, the norms $\|\cdot\|_{2, p(\cdot)},\|\cdot\|$ and $|\Delta(\cdot)|_{p(\cdot)}$ are equivalent. Furthermore in view of Proposition 2.2 , for every $u \in X$, we have

$$
\min \left\{\|u\|^{p^{+}},\|u\|^{p^{-}}\right\} \leq \int_{\Omega}|\Delta u|^{p(x)} d x \leq \max \left\{\|u\|^{p^{+}},\|u\|^{p^{-}}\right\} .
$$

Note that a weak solution for problem $\left(E_{\lambda}\right)$ satisfies the following definition. 
Definition 2.1. A function $u \in X$ is termed a weak solution of $\left(E_{\lambda}\right)$, if for all $v \in X$,

$$
\int_{\Omega}|\Delta u(x)|^{p(x)-2} \Delta u(x) \Delta v(x) d x=\int_{\Omega}\left(\lambda \frac{|u(x)|^{p(x)-2} u(x)}{\delta(x)^{2 p(x)}}+\mu|u(x)|^{p(x)-2} u\right) v(x) d x .
$$

If $u$ is not identically zero, then we say that $u$ is called an the eigenfunction of the $p(\cdot)$-biharmonic operator associated to the eigenvalue couple $(\lambda, \mu)$.

Definition 2.2. We call by the principal eigencurve of the $p(\cdot)$-biharmonic operator, the graph of the function $\lambda \rightarrow \mu_{1}(\lambda)$ from the interval $\left[0, C_{H}[\right.$ into $\mathbb{R}$ defined by:

$$
\mu_{1}(\lambda)=\inf \left\{\frac{\int_{\Omega} \frac{|\Delta u|^{p(x)}}{p(x)} d x-\lambda \int_{\Omega} \frac{1}{p(x)} \frac{|u|^{p(x)}}{\delta(x)^{2 p(x)}} d x}{\int_{\Omega} \frac{|u|^{p(x)}}{p(x)} d x} \mid u \in X \backslash\{0\}\right\} .
$$

Define the following functionals on $X$.

$$
\begin{aligned}
\Phi_{\lambda}(u) & =\int_{\Omega} \frac{1}{p(x)}|\Delta u|^{p(x)} d x-\lambda \int_{\Omega} \frac{1}{p(x)} \frac{|u|^{p(x)}}{\delta(x)^{2 p(x)}} d x \\
& =\Phi(u)-\lambda \varphi(u) .
\end{aligned}
$$

We set

$$
\mathcal{M}:=\left\{u \in X \mid \Psi_{p(\cdot)}(u)=1\right\} .
$$

Lemma 2.1. The following properties hold true:

(i) $\Phi_{\lambda}, \Psi_{p(\cdot)}$ and $\varphi$ are even, and of class $C^{1}$ on $X$.

(ii) $\mathcal{M}$ is a closed $C^{1}$-manifold.

Proof.

(i) It is clear that $\Phi_{\lambda}, \Psi_{p(.)}$ and $\varphi$ are even and of class $C^{1}$ on $X$.

(ii) $\mathcal{M}=\Psi_{p(.)}^{-1}(\{1\})$, therefore $\mathcal{M}$ is closed. Moreover, $\Psi_{p(.)}^{\prime}(u)$ is onto for all $u \in \mathcal{M}$. Indeed, let $u \in \mathcal{M}$ and consider the mapping

$$
f(t)=\Psi_{p(.)}(t u)=\int_{\Omega} \frac{1}{p(x)} t^{p(x)}|u|^{p(x)} d x, t \geq 0 .
$$

$f$ is differentiable for every $t>0$ and $f^{\prime}(t)=\left\langle\Psi_{p(.)}^{\prime}(t u), u\right\rangle>0$ since

$$
\left\langle\Psi_{p(.)}^{\prime}(t u), u\right\rangle=\int_{\Omega} t^{p(x)-1}|u|^{p(x)} d x \geq \min \left\{t^{p^{-}-1}, t^{p^{+}-1}\right\} \int_{\Omega}|u|^{p(x)} d x>\min \left\{t^{p^{-}-1}, t^{p^{+}-1}\right\} p^{-}>0
$$

Hence, $f$ is strictly increasing, further $f(0)=0$ and $\lim _{t \rightarrow \infty} f(t)=\infty$ since for $t$ large one has

$$
f(t) \geq \frac{1}{p^{+}} t^{p^{-}} \int_{\Omega}|u|^{p(x)} d x
$$


Thus for every $m \in \mathbb{R}$, there exists a unique $t_{0}>0$ such that $f\left(t_{0}\right)=m$, so that $\Psi_{p(.)}\left(t_{0} u\right)=m$ and we also have

$$
\left\langle\Psi_{p(.)}^{\prime}(u), \frac{t_{0} p(x)}{p(x)} u\right\rangle=\int_{\Omega}|u|^{p(x)-2} u \frac{t_{0}^{p(x)}}{p(x)} u d x=\int_{\Omega} \frac{1}{p(x)} t_{0} p(x)|u|^{p(x)} d x=\Psi_{p(.)}\left(t_{0} u\right)=m
$$

This means that $\Psi_{p(.)}^{\prime}(u)$ is onto for all $u \in \mathcal{M}$. Therefore $\Psi_{p(.)}$ is a submersion. Then $\mathcal{M}$ is a $C^{1}$-manifold of $X$.

Next, we write $\Phi_{\lambda}^{\prime}$ as

$$
\Phi_{\lambda}^{\prime}(.)=\Phi^{\prime}(.)-\lambda \varphi^{\prime}(.),
$$

where $\Phi^{\prime}, \varphi^{\prime}: X \rightarrow X^{*}$ are defined by

$$
\begin{gathered}
\left\langle\Phi^{\prime}(u), v\right\rangle:=\int_{\Omega}|\Delta u|^{p(x)-2} \Delta u \Delta v d x, \\
\left\langle\varphi^{\prime}(u), v\right\rangle:=\int_{\Omega} \frac{|u|^{p(x)-2}}{\delta(x)^{2 p(x)}} u v d x .
\end{gathered}
$$

Remark 2.2. Problem $\left(E_{\lambda}\right)$ can be equivalently written as

$$
\Phi_{\lambda}^{\prime}(u)=\mu \Psi_{p(.)}^{\prime}(u), u \in \mathcal{M},
$$

it is known that $(\mu, u)$ solves (2.5) if and only if $u$ is a critical point of $\Phi_{\lambda}$ with respect to $\mathcal{M}$.

We have the following properties about the operator $\Phi^{\prime}$.

Lemma 2.2 (see [6, Lemma 3.4]). The mapping $\Phi^{\prime}$ is continuous, bounded homeomorphism, strictly monotone, and of $\left(S_{+}\right)$type, namely

$$
u_{n} \rightarrow u \text { in } X \text { and } \varlimsup_{n \rightarrow \infty}\left\langle\Phi^{\prime}\left(u_{n}\right), u_{n}-u\right\rangle \leq 0 \text {, imply } u_{n} \rightarrow u \text { in } X .
$$

Lemma 2.3 (see [6, Lemma 3.5-(i)]). The functional $\varphi^{\prime}$ is sequentially weakly-strongly continuous, namely,

$$
u_{n} \rightarrow u \text { in } X \Longrightarrow \varphi^{\prime}\left(u_{n}\right) \rightarrow \varphi^{\prime}(u) \text { in } X^{*} \text {. }
$$

Lemma 2.4. The functional $\Psi_{p(.)}^{\prime}$ is sequentially weakly-strongly continuous.

Proof.

Let $u_{n} \rightarrow u$ in $X$. For any $v \in X$, by Hölder-type inequality (2.1) and continuous embedding $X \hookrightarrow L^{p(.)}(\Omega)$, it follows that

$$
\begin{aligned}
\left|\left\langle\Psi_{p(.)}^{\prime}\left(u_{n}\right)-\Psi_{p(.)}^{\prime}(u), v\right\rangle\right| & =\left|\int_{\Omega}\left(\left|u_{n}\right|^{p(x)-2} u_{n}-|u|^{p(x)-2} u\right) v d x\right| \\
& \leq\left. 2|| u_{n}\right|^{p(x)-2} u_{n}-\left.|u|^{p(x)-2} u\right|_{\frac{p(\cdot)}{p(\cdot)-1}}|v|_{p(\cdot)},
\end{aligned}
$$




$$
\leq\left. k_{1}|| u_{n}\right|^{p(x)-2} u_{n}-\left.|u|^{p(x)-2} u\right|_{\frac{p(\cdot)}{p(\cdot)-1}}\|v\|, \quad k_{1}>0
$$

On the other hand, using the compact embedding of $X$ into $L^{p(\cdot)}(\Omega)$, we have $u_{n} \rightarrow u$ in $L^{p(\cdot)}(\Omega)$. Thus,

$$
\left|u_{n}\right|^{p(x)-2} u_{n} \rightarrow|u|^{p(x)-2} u \quad \text { in } L^{\frac{p(\cdot)}{p(\cdot)-1}}(\Omega) .
$$

Lemma 2.5. For any $\lambda \in\left[0, C_{H}\left[\right.\right.$, the functional $\Phi_{\lambda}$ is bounded from below on $\mathcal{M}$.

Proof.

Let $u \in \mathcal{M}$. By (1.2), we have

$$
\frac{\lambda}{C_{H}} \int_{\Omega} \frac{1}{p(x)}|\Delta u|^{p(x)} d x \geq \lambda \int_{\Omega} \frac{1}{p(x)} \frac{|u|^{p(x)}}{\delta(x)^{2 p(x)}} d x .
$$

This implies that

$$
\Phi_{\lambda}(u) \geq\left(1-\frac{\lambda}{C_{H}}\right) \int_{\Omega} \frac{1}{p(x)}|\Delta u|^{p(x)} d x
$$

Thus

$$
\Phi_{\lambda}(u) \geq \lambda_{1}\left(1-\frac{\lambda}{C_{H}}\right)>-\infty,
$$

where $\mu_{1}(0)=\lambda_{1}$ is the first eigenvalue for the $p(\cdot)$-biharmonic operator, see [1].

Remark 2.3. We deduce from (2.7), that the eigenvalue $\mu_{1}$ is positive.

\section{Palais-Smale condition}

Proposition 3.1. The functional $\Phi_{\lambda}$ satisfies the Palais-Smale condition on $\mathcal{M}$, i.e., for $\left\{u_{n}\right\}_{n \geq 1} \subset \mathcal{M}$, if

$$
\left\{\Phi_{\lambda}\left(u_{n}\right)\right\}_{n} \text { is bounded, }
$$

and

$$
\alpha_{n}=\Phi_{\lambda}^{\prime}\left(u_{n}\right)-\beta_{n} \Psi_{p(.)}^{\prime}\left(u_{n}\right) \rightarrow 0 \text { as } n \rightarrow+\infty
$$

where

$$
\beta_{n}=\frac{\left\langle\Phi_{\lambda}^{\prime}\left(u_{n}\right), u_{n}\right\rangle}{\left\langle\Psi_{p(.)}^{\prime}\left(u_{n}\right), u_{n}\right\rangle}
$$

then $\left\{u_{n}\right\}_{n \geq 1}$ has a convergent subsequence in $X$.

Proof.

We have

$$
\begin{aligned}
\Phi\left(u_{n}\right)=\int_{\Omega} \frac{1}{p(x)}\left|\Delta u_{n}\right|^{p(x)} d x & \geq \frac{1}{p^{+}} \int_{\Omega}\left|\Delta u_{n}\right|^{p(x)} d x \\
& =\frac{1}{p^{+}} \rho_{p(.)}\left(\Delta u_{n}\right) .
\end{aligned}
$$


We know from (3.1) that $\left\{\Phi_{\lambda}\left(u_{n}\right)\right\}_{n}$ being bounded, and by (2.6) we have that $\rho_{p(.)}\left(\Delta u_{n}\right)$ is bounded in $\mathbb{R}$. Thus, without loss of generality, we can assume that

$$
u_{n} \text { converges weakly in } X \text { to a some function } u \in X \text {, }
$$

and

$$
\rho_{p(.)}\left(\Delta u_{n}\right) \rightarrow \ell
$$

For the rest we distinguish two cases:

Case 1. If $\ell=0$, then $u_{n} \rightarrow 0$ in $X$.

Case 2. If $\ell \neq 0$, let us prove that

$$
\overline{\lim }_{n \rightarrow \infty}\left\langle\Delta_{p(\cdot)}^{2} u_{n}, u_{n}-u\right\rangle \leq 0
$$

Indeed, notice that

$$
\left\langle\Delta_{p(\cdot)}^{2} u_{n}, u_{n}-u\right\rangle=\rho_{p(.)}\left(\Delta u_{n}\right)-\left\langle\Delta_{p(\cdot)}^{2} u_{n}, u\right\rangle .
$$

Applying $\alpha_{n}$ of (3.2) to $u$, we deduce that

$$
\theta_{n}=\left\langle\Delta_{p(\cdot)}^{2} u_{n}, u\right\rangle-\lambda\left\langle\varphi^{\prime}\left(u_{n}\right), u\right\rangle-\beta_{n}\left\langle\Psi_{p(.)}^{\prime}\left(u_{n}\right), u\right\rangle \rightarrow 0 \text { as } n \rightarrow \infty \text {. }
$$

Therefore

$$
\begin{aligned}
\left\langle\Delta_{p(\cdot)}^{2} u_{n}, u_{n}-u\right\rangle & =\rho_{p(.)}\left(\Delta u_{n}\right)-\lambda\left\langle\varphi^{\prime}\left(u_{n}\right), u\right\rangle-\theta_{n} \\
& -\frac{\left\langle\Phi_{\lambda}^{\prime}\left(u_{n}\right), u_{n}\right\rangle}{\left\langle\Psi_{p(.)}^{\prime}\left(u_{n}\right), u_{n}\right\rangle}\left\langle\Psi_{p(.)}^{\prime}\left(u_{n}\right), u\right\rangle .
\end{aligned}
$$

That is,

$$
\begin{aligned}
\left\langle\Delta_{p(.)}^{2} u_{n}, u_{n}-u\right\rangle & =\frac{\rho_{p(.)}\left(\Delta u_{n}\right)}{\left\langle\Psi_{p(.)}^{\prime}\left(u_{n}\right), u_{n}\right\rangle}\left(\left\langle\Psi_{p(.)}^{\prime}\left(u_{n}\right), u_{n}\right\rangle-\left\langle\Psi_{p(.)}^{\prime}\left(u_{n}\right), u\right\rangle\right) \\
& -\theta_{n}-\lambda\left\langle\varphi^{\prime}\left(u_{n}\right), u\right\rangle+\lambda \frac{\left\langle\varphi^{\prime}\left(u_{n}\right), u_{n}\right\rangle}{\left\langle\Psi_{p(.)}^{\prime}\left(u_{n}\right), u_{n}\right\rangle} \cdot\left\langle\Psi_{p(.)}^{\prime}\left(u_{n}\right), u\right\rangle .
\end{aligned}
$$

On one hand, according to Lemma 2.4, we deduce that

$$
\Psi_{p(.)}^{\prime}\left(u_{n}\right) \rightarrow \Psi_{p(.)}^{\prime}(u), \quad \text { and } \quad\left\langle\Psi_{p(.)}^{\prime}\left(u_{n}\right), u_{n}\right\rangle \rightarrow\left\langle\Psi_{p(.)}^{\prime}(u), u\right\rangle .
$$

By Lemma 2.3, we know that

$$
\varphi^{\prime}\left(u_{n}\right) \rightarrow \varphi^{\prime}(u), \quad\left\langle\varphi^{\prime}\left(u_{n}\right), u_{n}\right\rangle \rightarrow\left\langle\varphi^{\prime}(u), u\right\rangle \quad \text { and } \quad\left\langle\varphi^{\prime}\left(u_{n}\right), u\right\rangle \rightarrow\left\langle\varphi^{\prime}(u), u\right\rangle .
$$

Then

$$
\begin{aligned}
\left|\left\langle\Psi_{p(.)}^{\prime}\left(u_{n}\right), u_{n}\right\rangle-\left\langle\Psi_{p(.)}^{\prime}\left(u_{n}\right), u\right\rangle\right| & \leq\left|\left\langle\Psi_{p(.)}^{\prime}\left(u_{n}\right), u_{n}\right\rangle-\left\langle\Psi_{p(.)}^{\prime}(u), u\right\rangle\right| \\
& +\left|\left\langle\Psi_{p(.)}^{\prime}\left(u_{n}\right), u\right\rangle-\left\langle\Psi_{p(.)}^{\prime}(u), u\right\rangle\right| .
\end{aligned}
$$

It follows that

$$
\begin{aligned}
\left|\left\langle\Psi_{p(.)}^{\prime}\left(u_{n}\right), u_{n}\right\rangle-\left\langle\Psi_{p(.)}^{\prime}\left(u_{n}\right), u\right\rangle\right| & \leq\left|\left\langle\Psi_{p(.)}^{\prime}\left(u_{n}\right), u_{n}\right\rangle-\left\langle\Psi_{p(.)}^{\prime}(u), u\right\rangle\right| \\
& +\left.\left\|\Psi_{p(.)}^{\prime}\left(u_{n}\right)-\Psi_{p(.)}^{\prime}(u)\right\|\right|_{*}\|u\| .
\end{aligned}
$$


This implies that

$$
\left\langle\Psi_{p(.)}^{\prime}\left(u_{n}\right), u_{n}\right\rangle-\left\langle\Psi_{p(.)}^{\prime}\left(u_{n}\right), u\right\rangle \rightarrow 0 \text { as } n \rightarrow \infty .
$$

Combining with the above equalities, we obtain

$$
\varlimsup_{n \rightarrow+\infty}\left\langle\Delta_{p(.)}^{2} u_{n}, u_{n}-u\right\rangle \leq \frac{\ell}{\left\langle\Psi_{p(.)}^{\prime}(u), u\right\rangle} \varlimsup_{n \rightarrow \infty}\left(\left\langle\Psi_{p(.)}^{\prime}\left(u_{n}\right), u_{n}\right\rangle-\left\langle\Psi_{p(.)}^{\prime}\left(u_{n}\right), u\right\rangle\right) .
$$

We deduce

$$
\varlimsup_{n \rightarrow \infty}\left\langle\Delta_{p(\cdot)}^{2} u_{n}, u_{n}-u\right\rangle \leq 0
$$

On the other hand,

$$
\left\langle\Phi^{\prime}\left(u_{n}\right), u_{n}-u\right\rangle=\left\langle\Delta_{p(\cdot)}^{2} u_{n}, u_{n}-u\right\rangle .
$$

Then according to (3.4), we conclude that

$$
\varlimsup_{n \rightarrow \infty}\left\langle\Phi^{\prime}\left(u_{n}\right), u_{n}-u\right\rangle \leq 0 .
$$

In view of Lemma $2.2, u_{n} \rightarrow u$ strongly in $X$.

\section{Main results}

In this section we prove the existence of at least one non-decreasing sequence of eigencurves to problem $\left(E_{\lambda}\right)$. Our approach is based on Ljusternik-Schnirelmann theory on $C^{1}$-manifolds [13].

Let us now state our first main result of this paper.

Theorem 4.1. For any $\lambda \in\left[0, C_{H}\left[\right.\right.$, the problem $\left(E_{\lambda}\right)$ has at least one non-decreasing sequence of eigencurves $\left(\mu_{j}\right)_{j \geq 1}$ given by

$$
\mu_{j}(\lambda):=\inf _{K \in \Gamma_{j}} \max _{u \in K} \Phi_{\lambda}(u) \rightarrow \infty, \text { as } j \rightarrow \infty,
$$

where

$$
\Gamma_{j}:=\{K \subset \mathcal{M} \mid K \text { is compact, } K=-K \text { and } \gamma(K) \geq j\},
$$

here $\gamma(K)$ indicates the genus of $K$.

We start with two auxiliary results.

Lemma 4.1. For any $j \in \mathbb{N}^{*}, \Gamma_{j} \neq \varnothing$.

Proof.

Since $X$ is separable. Therefore, for any $j \in \mathbb{N}^{*}$, there exists $\left(\varphi_{i}\right)_{i \geq 1}$ linearly dense in $X$ such that

$$
\left\{\begin{array}{l}
\operatorname{supp}\left(\varphi_{i}\right) \cap \operatorname{supp}\left(\varphi_{j}\right)=\varnothing \text { if } i \neq j, \\
\operatorname{meas}\left(\operatorname{supp}\left(\varphi_{i}\right)\right)>0 \text { for } i \in\{1,2, \ldots, j\} .
\end{array}\right.
$$

Let $X_{j}=\operatorname{Span}\left\{\varphi_{1}, \varphi_{2}, \ldots, \varphi_{j}\right\}$ be the vector subspace of $X$ generated by $j$ vectors $\left\{\varphi_{1}, \varphi_{2}, \ldots, \varphi_{j}\right\}$, then $\operatorname{dim} X_{j}=j$. 
Note that $X_{j} \subset L^{p(\cdot)}(\Omega)$ because $X_{j} \subset X \subset L^{p(\cdot)}(\Omega)$. Thus the norm $\|\cdot\|$ and $|\cdot|_{p(\cdot)}$ are equivalent on $X_{j}$ because $X_{j}$ is a finite dimensional space. Consequently the map

$$
u \mapsto|u|_{p(\cdot)}:=\inf \left\{\alpha>0: \int_{\Omega}\left|\frac{u(x)}{\alpha}\right|^{p(x)} d x \leq 1\right\},
$$

defines a norm on $X_{j}$. Denote $S:=\left\{u \in X_{j}:|u|_{p(\cdot)}=1\right\}$ the unit sphere of $X_{j}$.

Let us introduce the functional

$$
\begin{aligned}
& g: \mathbb{R}^{+} \times X_{j} \longrightarrow \mathbb{R} \\
& (\xi, u) \mapsto \Psi_{p(\cdot)}(\xi u) .
\end{aligned}
$$

On one hand, it is clear that

- $g(0, u)=0$.

- $g(\xi, u)$ is non-decreasing with respect to $\xi$.

Moreover, for $\xi>1$ we have

$$
g(\xi, u) \geq \xi^{p^{-}} \Psi_{p(\cdot)}(u),
$$

so that $\lim _{\tilde{\zeta} \rightarrow+\infty} g(\xi, u)=+\infty$. Therefore, for every $u \in S$ fixed, there is a unique value $\xi=\xi(u)>0$ such that $g(\xi(u), u)=1$.

On the other hand, since

$$
\frac{\partial g}{\partial \xi}(\xi(u), u)=\int_{\Omega}(\xi(u))^{p(x)-1}|u|^{p(x)} d x \geq \frac{p^{-}}{\xi(u)} g(\xi(u), u)=\frac{p^{-}}{\xi(u)}>0 .
$$

The implicit function theorem implies that the map $u \mapsto \xi(u)$ is continuous and even by uniqueness. Now, take the compact $H_{j}:=\mathcal{M} \cap X_{j}$. Since the map $h: S \longrightarrow H_{j}$ defined by $h(u)=\xi(u) \cdot u$ is continuous and odd, it follows by the property of genus that $\gamma\left(H_{j}\right)=j$. Therefore $H_{j} \in \Gamma_{j}$.

\section{Lemma 4.2.}

$$
\mu_{j}(\lambda) \rightarrow \infty \text { as } j \rightarrow \infty
$$

\section{Proof.}

Let $\left(e_{k}, e_{n}^{*}\right)_{k, n}$ be a bi-orthogonal system satisfying

- $\left(e_{k}\right)_{k}$ are linearly dense in $X$,

- $\left(e_{n}^{*}\right)_{n}$ total for $X^{*}$,

- $\left\langle e_{n}^{*}, e_{k}\right\rangle=0$ if $n \neq k$,

- $\left\langle e_{n}^{*}, e_{n}\right\rangle=1, \forall n \in \mathbb{N}^{*}$.

Set for every $k \in \mathbb{N}^{*}$,

$$
F_{k}:=\operatorname{span}\left\{e_{1}, \ldots, e_{k}\right\} \quad \text { and } F_{k}^{\perp}:=\operatorname{span}\left\{e_{k+1}, e_{k+2}, \ldots\right\} .
$$

By the property of genus, we have for any $K \in \Gamma_{k}$,

$$
K \cap F_{k-1}^{\perp} \neq \varnothing \text {. }
$$

Let us prove that

$$
t_{k}:=\inf _{K \in \Gamma_{k}} \sup _{u \in K \cap F_{k-1}^{\perp}} \Phi_{\lambda}(u) \rightarrow \infty \text { as } k \rightarrow \infty .
$$


Arguing by contradiction. Assume that for $k$ is large enough, there exists $u_{k} \in F_{k-1}^{\perp}$ with $\int_{\Omega} \frac{\mid u_{k} p^{(x)}}{p(x)} d x=1$ such that

$$
t_{k} \leq \Phi_{\lambda}\left(u_{k}\right) \leq M
$$

for some constant $M>0$ independent on $k$. Thus in view of (2.6), we get

$$
\left\|u_{k}\right\| \leq\left(\frac{p^{+} C_{H} M}{C_{H}-\lambda}\right)^{\frac{1}{p^{-}}}
$$

This implies that $\left(u_{k}\right)_{k}$ is bounded in $X$. We may assume that $u_{k} \rightarrow u$ in $X$ and $u_{k} \rightarrow u$ in $L^{p(\cdot)}(\Omega)$. By our choice of $F_{k-1}^{\perp}$, we observe that $u_{k} \rightarrow 0$ in $X$ because $\left\langle e_{n}^{*}, e_{k}\right\rangle=0$, for any $k>n$. This contradicts the fact that $1=\int_{\Omega} \frac{\left|u_{k}\right| p(x)}{p(x)} d x \rightarrow 0$ for every $k$. Hence $\lambda_{k} \geq t_{k}$, for every $k \geq 1$.

Proof of Theorem 4.1. Applying lemmas 4.1, 4.2 and Ljusternik-Schnirelemann theory to the problem $\left(E_{\lambda}\right)$, we have for each $j \in \mathbb{N}^{*}, \mu_{j}(\lambda)$ is a critical value of $\left(E_{\lambda}\right)$ on $C^{1}$-manifold $\mathcal{M}$, such that

$$
\mu_{j}(\lambda) \rightarrow \infty \text {, as } j \rightarrow \infty .
$$

Corollary 4.1. The following statements hold true:

(i) $\mu_{1}(\lambda)=\inf \left\{\frac{\int_{\Omega} \frac{1}{p(x)}|\Delta u|^{p(x)} d x-\lambda \int_{\Omega} \frac{1}{p(x)} \frac{\mid u u^{p(x)}}{\delta(x)^{2 p(x)}} d x}{\int_{\Omega} \frac{1}{p(x)}|u|^{p(x)} d x} \mid u \in X \backslash\{0\}\right\}$.

(ii) $0<\mu_{1}(\lambda) \leq \mu_{2}(\lambda) \leq \cdots \leq \mu_{n}(\lambda) \rightarrow+\infty$.

Proof.

(i) For $u \in \mathcal{M}$, set $K_{1}=\{u,-u\}$. It is clear that $\gamma\left(K_{1}\right)=1, \Phi_{\lambda}$ is even and

$$
\Phi_{\lambda}(u)=\max _{K_{1}} \Phi_{\lambda} \geq \inf _{K \in \Gamma_{1}} \max _{u \in K} \Phi_{\lambda}(u) .
$$

Thus

$$
\inf _{u \in \mathcal{M}} \Phi_{\lambda}(u) \geq \inf _{K \in \Gamma_{1}} \max _{u \in K} \Phi_{\lambda}(u)=\mu_{1}(\lambda)
$$

On the other hand, for all $K \in \Gamma_{1}$ and $u \in K$, we have

$$
\max _{u \in K} \Phi_{\lambda} \geq \Phi_{\lambda}(u) \geq \inf _{u \in \mathcal{M}} \Phi_{\lambda}(u)
$$

It follows that

$$
\inf _{K \in \Gamma_{1}} \max _{K} \Phi_{\lambda}=\mu_{1}(\lambda) \geq \inf _{u \in \mathcal{M}} \Phi_{\lambda}(u)
$$

Then

$$
\mu_{1}(\lambda)=\inf \left\{\frac{\int_{\Omega} \frac{1}{p(x)}|\Delta u|^{p(x)} d x-\lambda \int_{\Omega} \frac{1}{p(x)} \frac{\mid u p^{p(x)}}{\delta(x)^{2 p(x)}} d x}{\int_{\Omega} \frac{1}{p(x)}|u|^{p(x)} d x} \mid u \in X \backslash\{0\}\right\} .
$$

(ii) For all $i \geq j$, we have $\Gamma_{i} \subset \Gamma_{j}$ and in view of the definition of $\lambda_{i}, i \in \mathbb{N}^{*}$, we get $\mu_{i}(\lambda) \geq \mu_{j}(\lambda)$. As regards $\mu_{n}(\lambda) \rightarrow \infty$, it has been proved in Theorem 4.1. 
Author Contributions. The corresponding author is the only author responsible for the writing and revision of this manuscript.

Acknowledgements. The authors would like to thank the referees for their careful reading and insightful comments of the manuscript.

\section{References}

[1] A. Ayoujil, A. EI Amrouss, On the spectrum of a fourth order elliptic equation with variable exponent, Nonlinear Anal. Theory Methods., 71(2009), 4916-4926.

[2] P.A. Binding, Y.X. Huang, Bifurcation from eigencurves of the p-Laplacian, Differential and Integral Equations., 8(2)(1995), 405-414.

[3] L. Diening, P. Harjulehto, P. Hästö, M. Růžička, Lebesgue and Sobolev Spaces with Variable Exponents, SpringerVerlag, Heidelberg., (2011).

[4] A. El khalil, On the spectrum of Robin boundary p-Laplacian problem, Moroccan J. of Pure and Appl. Anal.(MJPAA)., 5(2) (2019), 279-293.

[5] A. El khalil, S. Kellati, A. Touzani, On the principal frequency curve of the p-biharmonic operator, Arab journal of Mathematical Sciences., 17 (2011), 89-99.

[6] A. El khalil, M. El Moumni, M.D. Morchid Alaoui, A. Touzani, $p(x)$-Biharmonic operator involving $p(x)$-Hardy's inequality, Georgian Mathematical Journal., DOI: https://doi.org/10.1515/gmj-2018-0013

[7] Abdelouahed El khalil, Mohamed Laghzal, Moulay Driss Morchid Alaoui, Abdelfattah Touzani, Eigenvalues for a class of singular problems involving $p(x)$-Bihamonic operator and $q(x)$-Hardy potential, Adv. Nonlinear Anal., 9 (2020), 1130-1144.

[8] A. El khalil, M.D. Morchid Alaoui, M. Laghzal, A. Touzani, On a nonlinear PDE involving weighted p-Laplacian, Bol. Soc. Paran. Mat., 38 (2020), 131-145.

[9] A. El khalil, M.D. Morchid Alaoui, A. Touzani, On the spectrum of the p-biharmonic operator involving p-Hardy's inequality, Appl. Math. Warsaw., 41 (2014), 239-246.

[10] X.L. Fan, X. Fan, A Knobloch-type result for $p(t)$-Laplacian systems, J. Math. Anal. Appl., 282 (2003), 453-464.

[11] X.L. Fan, D. Zhao, On the spaces $L^{p(x)}(\Omega)$ and $W^{m, p(x)}(\Omega)$, J. Math. Anal. Appl., 263 (2001), 424-446.

[12] M. Mihăilescu, Existence and multiplicity of solutions for a Neumann problem involving the $p(x)$-Laplace operator, Nonlinear Anal., 67 (2007), 1419-1425.

[13] A. Szulkin, Ljusternik-Schnirelmann theory on $C^{1}$-manifolds, Ann. Inst. Henri Poincaré Anal. Nonl., 5(2) (1988), 119-139.

[14] A. Zang, Y. Fu, Interpolation inequalities for derivatives in variable exponent Lebesgue-Sobolev spaces, Nonlinear Anal., 69 (2008), 3629-3636. 